\title{
The Prognostic Value of the C-reactive Protein/Prognostic Nutritional Index Ratio in Stage III and IV Laryngeal Cancer Patients Treated with Radiotherapy
}

Jie $\mathrm{Fu}^{1}$, Xiaojing Yang ${ }^{1}$

1. Radiation Oncology, Shanghai Jiao Tong University Affliated Sixth People’s Hospital, Shanghai, CHN

Corresponding author: Xiaojing Yang, youngshanghai@126.com

\section{Abstract}

\section{Objective}

Patients with advanced laryngeal cancer have a poor prognosis. The purpose of this work was to analyze the connection between clinical factors and survival and to try to identify survival prognostic factors.

\section{Methods}

Sixty-one laryngeal cancer patients received radiotherapy. All of their clinicopathologic material was gathered from a retrospective review of the medical records and subjected to further analysis. We examined the prognostic significance of the C-reactive protein $(\mathrm{CRP})$ /prognostic nutritional index $(\mathrm{PNI})$ ratio.

\section{Results}

We assessed the CRP and PNI levels before radiotherapy. An analysis of overall survival (OS) of patients with high CRP/PNI was markedly shorter than for those patients with a low CRP/PNI $(\mathrm{P}=0.010)$. Multivariable analysis showed that a high CRP/PNI ratio was a disadvantageous independent prognostic marker.

\section{Conclusions}

The data show that CRP/PNI may be used as a prognostic indicator for laryngeal cancer patients treated with radiotherapy.

Received 04/10/2019

Review began 04/12/2019 Review ended 05/08/2019

Published 05/12/2019

\section{(C) Copyright 2019}

Fu et al. This is an open access article distributed under the terms of the Creative Commons Attribution License CC-BY 3.0., which permits unrestricted use, distribution, and reproduction in any medium, provided the original author and source are credited.
Categories: Oncology

Keywords: laryngeal cancer, crp/pni, prognosis

\section{Introduction}

Laryngeal cancer is a common malignancy of the head and neck. The incidence of laryngeal cancer is notably higher in men than in women [1]. The most common pathological type of larynx cancer, making up the majority of cases, is squamous cell cancer [2]. The treatment of laryngeal cancer usually depends on surgical treatment and radiotherapy. Radiotherapy plays an important role in patients at advanced clinical stages [3]. Although treatment methods continue to improve, the therapeutic effect is still not satisfactory. The establishment of a simple and convenient marker system to help determine the prognosis of patients with

How to cite this article

Fu J, Yang X (May 12, 2019) The Prognostic Value of the C-reactive Protein/Prognostic Nutritional Index Ratio in Stage III and IV Laryngeal Cancer Patients Treated with Radiotherapy. Cureus 11(5): e4648. DOI 10.7759 /cureus.4648 
laryngeal cancer would be of great significance.

There are reports showing that C-reactive protein (CRP) levels are associated with inflammatory responses. High expression of CRP indicates a poor outcome in patients with malignant tumors, including laryngeal cancer [4]. In addition, it has also been proposed that the inflammatory response is pathogenic to cancer-related malnutrition [5-6]. Malnutrition, in turn, is associated with poor overall state, shortened survival, and increased mortality in cancer patients [7]. The prognostic nutrition index (PNI) is used to evaluate the outcomes of patients with malignant tumors, such as esophageal cancer [8], lung cancer [9], and gastric cancer [10]. In recent work, CRP/PNI showed a prognostic ability in esophageal cancer patients [8] and fracture surgery patients [11]. Raised CRP/PNI predicted poor prognosis. However, there has been no research that has shown the relationship between the prognostic value of CRP/PNI and laryngeal cancer.

The object of this research was to evaluate the significance of CRP/PNI and survival in laryngeal cancer patients. We looked into the impact of CRP/PNI on overall survival (OS) in laryngeal cancer and contrasted the predictive value of platelet count, CRP, PNI, leukocytosis, lymphocyte counts, anemia, and CRP/PNI.

\section{Materials And Methods}

\section{Patients and data collection}

We retrospectively analyzed 61 patients with laryngeal cancer who were treated in our department from January 2009 to June 2014. The main standards for inclusion are as follows: (i) all of the patients had a pathological diagnosis of laryngeal cancer; and (ii) none of the patients had undergone chemotherapy or radiotherapy before. Patients were excluded for the subsequent criteria: (i) acute infection occurring within two weeks; (ii) other blood system diseases; or (iii) incomplete data. The following clinical parameters were recorded: gender, age at diagnosis, differentiation, tumor stage, tumor, nodes, and metastases (TNM) stage, lymph node metastasis (LNM), and smoking history. The laboratory characteristics of blood reports, including platelet count, CRP, PNI, leukocytosis, lymphocyte counts, and anemia, were performed before radiotherapy. The following formula was calculated for PNI: 0.05 total lymphocyte count $(/ \mathrm{mm} 3)+10 \times$ serum albumin $(\mathrm{g} / \mathrm{dL})$ [12]. Authors have access to information that could identify individual participants during or after data collection. Ethical approval for the use of patient tissues was provided by the Ethics Committee of Shanghai Sixth People's Hospital (Shanghai, China). Informed consent was obtained from all patients.

\section{Statistical analysis}

The data analysis was performed using the SPSS statistical software package (Version 20.0; IBM Corporation, Armonk, NY, USA). The Spearman rank correlation survival analysis was used to study the correlation between CRP and PNI. The survival analysis was tested for significance using Kaplan-Meier curves and log-rank tests. Univariable Cox regression analyses were performed using death as the outcome. The Cox proportional hazards model was used for multivariate analysis, to evaluate the relationship between CRP/PNI and other prognostic factors and OS. We calculated the hazard ratio and $95 \%$ confidence interval. $\mathrm{P}<0.05$ was considered statistically significant.

\section{Results}

\section{Patient characteristics}

Sixty-one patients undergoing radiotherapy were involved in this study. Each patient's features are shown in Table 1 . The median age of the patients was 57.2 years $( \pm 7.12)$ and 59 patients 


\section{Cureus}

(96.7\%) were male. The patients' tumor stage distributed from T1 to T4, and there were 27 (44.3\%) patients with negative N-stage. Among the 61 patients, $46(75.4 \%)$ had a history of smoking. The cell types in our data showed 59 patients $(96.7 \%)$ with squamous cell carcinoma and the others were adenocarcinoma.

\section{Characteristics}

Gender

Male

Female

Age, years

Mean

SD

Differentiation

Well/moderate

Poor/undifferentiation

Tumor stage

T1

T2

T3

T4

Tumor, node, metastases (TNM) stage

III

$35(57.4)$

IV

Lymph node metastasis (LNM)

Yes

No

$34(55.7)$

Smoking history

Yes

$46(75.4)$

No

$15(24.6)$

Pack-years

$\leq 30$

$>30$
$26(42.6)$

$35(57.4)$ 


\section{Cureus}

Survival status

Dead

Alive

$25(41.0)$

TABLE 1: Clinicopathological characteristics of laryngeal cancer patients

\section{CRP/PNI ratio as a prognostic marker of survival}

The mean values of CRP and PNI were $13.65 \pm 7.34 \mathrm{mg} / \mathrm{L}$ and $38.42 \pm 8.26$, respectively. There was a negative correlation between PNI and CRP with a coefficient of -0.741 (Figure 1; $\mathrm{p}=0.047$ ). We show the relationship between CRP/PNI ratios and clinical properties in Table 2 . The cut-off value of the CRP/PNI ratio was 0.10 according to the receiver operating characteristic (ROC) analysis. According to this value, patients were separated into the CRP/PNI ratio $\leqslant 0.10$ group and the CRP/PNI ratio $>0.10$ group. Twelve patients $(19.7 \%)$ had a CRP/PNI ratio of $\leqslant 0.10$ and 49 patients $(80.3 \%$ ) had a CRP/PNI ratio of $>0.10$. The CRP/PNI ratio was associated with CRP $(\mathrm{p}<0.001)$, PNI $(\mathrm{p}<0.001)$, platelet count $(\mathrm{p}=0.049)$, hypoalbuminemia $(\mathrm{p}=0.032)$, lymphocyte count $(\mathrm{p}=0.046)$, and survival $(\mathrm{p}=0.010)$. However, no relationship was observed between the CRP/PNI ratio and other factors, such as comorbidities, leukocytosis, and anemia. Kaplan-Meier survival curves with high CRP ( $<<0.001$, Figure $2 A)$ or low PNI $(p<0.001$, Figure $2 B$ ) were associated with poor survival. In addition, patients with a low proportion of CRP/PNI and a high CRP/PNI ratio displayed obvious separation ( $\mathrm{p}=0.027$, Figure 2C).

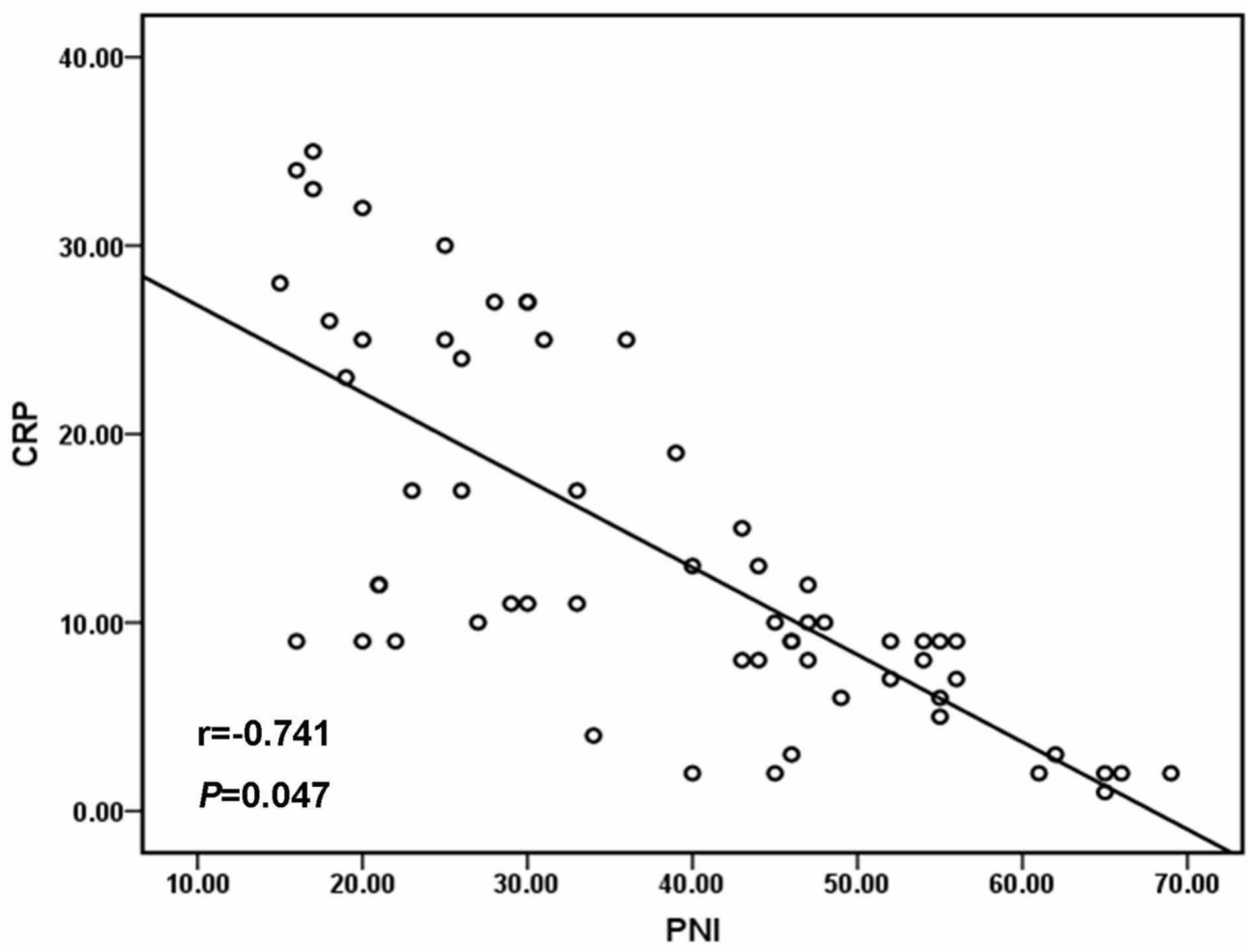

\section{FIGURE 1: Pearson correlation}

A significant negative correlation between CRP and PNI $(r=-0.741, p=0.047)$ 


\section{Cureus}

$\begin{array}{ll} & \text { Tot } \\ \text { Age (years) } & 57 \pm 7 \\ \text { Men } & 59 \\ \text { Female } & 2 \\ \text { CRP, mg/L } & \\ <10 & 31 \\ \geq 10 & 30\end{array}$

\section{CRP/PNI ratio}

p-value

$57 \pm 7$

30

33

$<214$

$\geq 214$

28

40

21

10

30

0.190

No

Anemia

Yes

31

No

Lymphocyte counts, $/ \mathrm{mm}^{3}$

$<1673$

$\geq 1673$

28

Hypoalbuminemia

Yes

No

29

PNI

$<44$

$\geq 44$

Survival status

Dead

$$
\leq 0.10 \quad>0.10
$$

$\begin{array}{lll}56 \pm 3 & 58 \pm 6 & 0.317\end{array}$

11

48

0.752

1

19

$<0.001^{*}$

30

$0 \quad 30$

3

30

$0.049^{*}$

19

19

2

25

0.949

24

29

$0.046^{*}$

20

$8 \quad 20$

2

4

28

$0.032^{*}$

$8 \quad 21$

34

1

33

$<0.001^{*}$

27

11

16 


\section{Cureus}

Alive

\section{TABLE 2: CRP/PNI ratio and clinicopathological parameters}

$\mathrm{CRP}=\mathrm{C}$-reactive protein, $\mathrm{PNI}=$ prognostic nutritional index, ${ }^{*} p<0.05$ is considered significant
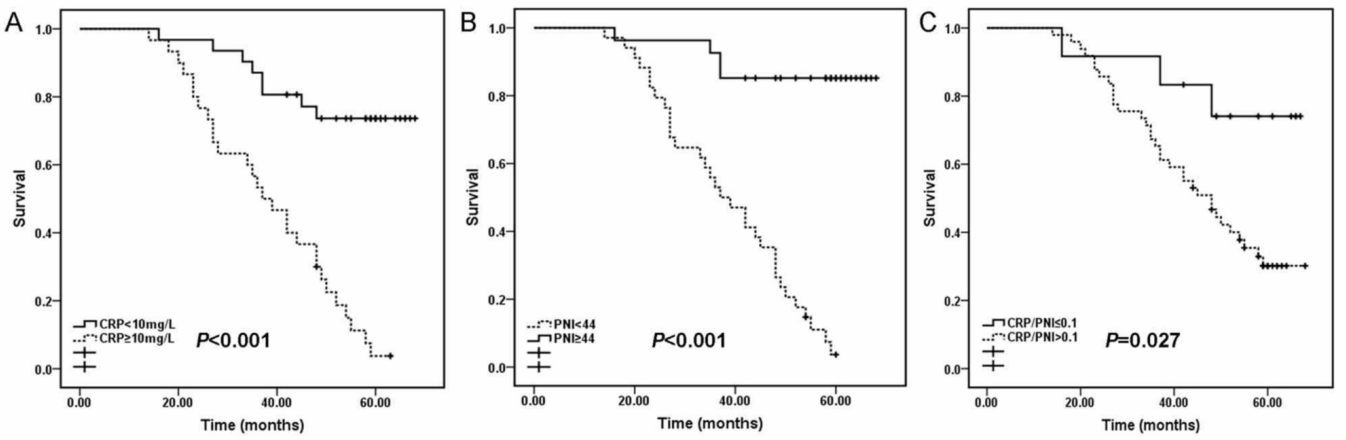

\section{FIGURE 2: Kaplan-Meier survival curves stratified by CPR, PNI,} and CRP/PNI ratio

A and B: patients with elevated CRP $(p<0.001)$ or decreased PNI $(p<0.001)$ were associated with decreased five-year survival, respectively. C: Patients with a CRP/PNI ratio of $\leq 0.10$ had better survival than patients with a CRP/PNI ratio of $>0.10(p<0.001)$.

CRP: C-reactive protein, PNI: prognostic nutritional index

\section{Survival and prognostic factor analysis}

The five-year OS value was $41.0 \%$. The univariate and multivariate analyses of OS in all the patients are shown in Table 3 and Table 4, respectively. In the univariate analysis, PNI $(p<0.001), \operatorname{CRP}(p=0.04)$, lymphocyte counts $(p<0.001)$, leukocytosis $(p=0.015)$, hypoalbuminemia ( $\mathrm{p}=0.037)$, and CRP/PNI ( $\mathrm{p}=0.010)$ were found to have a significant impact on OS. In the multivariate analysis, we found PNI $(p=0.009)$ and CRP/PNI $(p=0.018)$ to be predictive factors for survival. Collectively, these data show that a high CRP/PNI ratio predicts poor prognosis for laryngeal cancer.

\begin{tabular}{|c|c|c|c|c|}
\hline & \multirow[t]{2}{*}{ Total cases (n) } & \multicolumn{2}{|c|}{ Survival status } & \multirow[t]{2}{*}{$p$-value } \\
\hline & & Dead=36 & Alive=25 & \\
\hline Age (years) & $57 \pm 7$ & $58 \pm 3$ & $56 \pm 2$ & 0.065 \\
\hline Men & 59 & 36 & 23 & 0.071 \\
\hline Female & 2 & 0 & 2 & \\
\hline \multicolumn{5}{|l|}{ CRP, mg/L } \\
\hline$<10$ & 31 & 8 & 23 & $<0.001^{*}$ \\
\hline
\end{tabular}




\section{Cureus}

Platelet count, $/ \mathrm{mm}^{3}$

$<214$

$\geq 214$

Leukocytosis

Yes

40

21

31

30

33

28

$\geq 1673$

Hypoalbuminemia

Yes

32

29

34

27

32

4

2

$<0.001^{*}$

$\geq 44$

CRP/PNI

$\leq 0.10$

12

$>0.10$

49

14

22

12

24

25

11

18

23

TABLE 3: Survival status and clinicopathological parameters in 61 specimens

$\mathrm{CRP}=\mathrm{C}$-reactive protein, $\mathrm{PNI}=$ prognostic nutritional index, ${ }^{*} \mathrm{p}<0.05$ is considered significant 


\section{Cureus}

\begin{tabular}{|c|c|c|c|}
\hline & Hazard ratio & $95 \% \mathrm{Cl}$ & $\mathbf{P}$ \\
\hline Age (years) & 1.578 & $0.870 \sim 2.518$ & 0.570 \\
\hline Sex & 1.503 & $0.358 \sim 3.611$ & 0.145 \\
\hline CRP, mg/L & 2.577 & $0.729 \sim 7.961$ & 0.052 \\
\hline PNI & 0.143 & $0.076 \sim 8.047$ & $0.009^{*}$ \\
\hline Platelet count, $/ \mathrm{mm}^{3}$ & 1.937 & $0.722 \sim 5.741$ & 0.198 \\
\hline Leukocytosis & 1.332 & $0.544 \sim 4.902$ & 0.731 \\
\hline Anemıa & 1. 476 & $0.786 \sim 4.516$ & 0.415 \\
\hline Lymphocyte counts, $/ \mathrm{mm}^{3}$ & 2.726 & $1.9013 \sim 7.457$ & 0.062 \\
\hline Hypoalbuminemia & 3.114 & 1. 731 5.920 & 0.326 \\
\hline CRP/PNI & 2.375 & $2.104 \sim 11.101$ & $0.018^{x}$ \\
\hline
\end{tabular}

\section{TABLE 4: Contribution of various potential prognostic factors to survival by Cox regression analysis in 61 specimens}

$\mathrm{CRP}=\mathrm{C}$-reactive protein, $\mathrm{PNI}=$ prognostic nutritional index, $\mathrm{Cl}=$ confidence interval, Statistical analyses were performed by the logrank test, ${ }^{*} p<0.05$ is considered significant

\section{Discussion}

Therapeutic effects on patients with advanced laryngeal cancer have not significantly improved in the past two decades [13]. Our study aimed to discover a novel, comprehensive, and economical index for prognostic prediction. In this research, we showed that the CRP/PNI ratio was a predictor of advanced laryngeal cancer patients.

The occurrence and development of cancer are closely related to inflammation [14]. CRP is an important inflammatory marker whose levels rise in response to infection or trauma [15]. It has been reported that high levels of CRP can promote tumorigenesis and lead to poor prognosis in ovarian cancer [16-17], colon cancer [18], glioblastoma [19], and other conditions. CRP can increase vascular growth factors levels and interleukins in the peripheral blood, thereby promoting the formation of tumor blood vessels [20]. In the present study, patients with lower CRP level ( $\leqslant 10.0 \mathrm{mg} / \mathrm{L}$ ) had better survival rates than patients with CRP $>10.0 \mathrm{mg} / \mathrm{L}$ (74.2\% vs. $6.7 \%, \mathrm{p}<0.001)$. Unfortunately, in multivariate analysis, there was no evidence that CRP is an independent prognostic factor $(p=0.052)$.

More than $20 \%$ of cancer patients are reported to die of malnutrition rather than the tumor itself [21]. Laryngeal cancer patients are more prone to malnutrition due to the tumor mass and dysphagia [22]. PNI is calculated from serum albumin and lymphocyte counts. Albumin is closely related to inflammatory responses in cancer patients [23]. Lymphocytes regulate the immunologic damage caused by tumor cells and take a significant part in the immune response [24]. PNI is related to the prognosis of cancer patients with esophageal carcinoma [25], nonsmall cell lung cancer [26], ovarian cancer [27], and hepatocellular carcinoma [7]. PNI is 
considered an independent marker of poor prognosis in cancer patients. Consistent with previous results, PNI was found to be an independent prognostic factor in our results $(\mathrm{p}=0.009)$.

A single indicator may be affected by many factors; the ratio of CRP and PNI may reduce this effect. The ratio of CRP/PNI is more predictive than CRP or PNI by themselves. Thus, in the present research, we first studied the importance of CRP/PNI in evaluating the prognosis of laryngeal cancer patients. Compared with a CRP/PNI ratio of $>0.10$, patients with a CRP/PNI ratio of $\leqslant 0.10$ had a better survival prognosis $(\mathrm{p}=0.001$ ). In a multivariate analysis, the CRP/PNI ratio is an important non-independent prognostic factor $(\mathrm{p}=0.018)$. Consistent with previous results in esophageal cancer, our data show that the CRP/PNI ratio could be a predictor of laryngeal cancer.

This work has some specific deficiencies. First, our results are consistent with the predecessor's outcomes. We found the CRP/PNI ratio to be a prognostic indicator. However, inflammatory marker leukocytosis is also related to survival in our results. The CRP/PNI ratio should be assessed together with leukocytosis in laryngeal cancer patients in further studies. What is more important is that this is a retrospective analysis of only 61 advanced laryngeal cancer cases. The sample size of the study is not sufficient. In future studies, a larger cohort of patients is required. If conditions permit, we hope to conduct a prospective study to enable a better evaluation of prognostic factors for laryngeal cancer patients.

In summary, our study identified a CRP/PNI ratio of $>0.10$ to be a signature indicator for the outcomes of advanced laryngeal cancer. The potential role of the CRP/PNI ratio and other indicators in laryngeal cancer remains to be investigated at a broader and deeper level.

\section{Conclusions}

The purpose of our work is to analyze the connection between clinical factors and survival and to try to identify survival prognostic factors. We examined the prognostic significance of the Creactive protein (CRP)/prognostic nutritional index (PNI) ratio. CRP/PNI may be used as a prognostic indicator for laryngeal cancer patients treated with radiotherapy.

\section{Additional Information \\ Disclosures}

Human subjects: Consent was obtained by all participants in this study. Ethics Committee of Shanghai Sixth People's Hospital issued approval 2018-95-(2). Ethical approval for the use of patient tissues was provided by the Ethics Committee of Shanghai Sixth People's Hospital (Shanghai, China). Animal subjects: All authors have confirmed that this study did not involve animal subjects or tissue. Conflicts of interest: In compliance with the ICMJE uniform disclosure form, all authors declare the following: Payment/services info: All authors have declared that no financial support was received from any organization for the submitted work. Financial relationships: All authors have declared that they have no financial relationships at present or within the previous three years with any organizations that might have an interest in the submitted work. Other relationships: All authors have declared that there are no other relationships or activities that could appear to have influenced the submitted work.

\section{Acknowledgements}

We thank LetPub (www.letpub.com) for its linguistic assistance during the preparation of this manuscript.

\section{References}


1. Pantel M, Guntinas-Lichius O: Laryngeal carcinoma: epidemiology, risk factors and survival [Article in German]. HNO. 2012, 60:32-40. 10.1007/s00106-011-2423-8

2. Licitra L, Bernier J, Grandi C, Locati L, Merlano M, Gatta G, Lefebvre JL: Cancer of the larynx. Crit Rev Oncol Hematol. 2003, 47:65-80. 10.1016/S1040-8428(03)00017-9

3. Nelke KH, Pawlak W, Leszczyszyn J, Gerber H: Photodynamic therapy in head and neck cancer. Postepy Hig Med Dosw (Online). 2014, 68:119-28. 10.5604/17322693.1088044

4. Zeng YC, Xue M, Chi F, et al.: C-reactive protein level predicts prognosis in patients with locoregionally advanced laryngeal carcinoma treated with chemoradiotherapy. Tumour Biol. 2012, 33:891-895. 10.1007/s13277-012-0330-6

5. Argiles JM, Busquets S, Lopez-Soriano FJ: Cytokines in the pathogenesis of cancer cachexia . Curr Opin Clin Nutr Metab Care. 2003, 6:401-406. 10.1097/01.mco.0000078983.18774.cc

6. Argiles JM, Moore-Carrasco R, Fuster G, Busquets S, Lopez-Soriano FJ: Cancer cachexia: the molecular mechanisms. Int J Biochem Cell Biol. 2003, 35:405-409. 10.1016/S1357-

2725(02)00251-0

7. Pinato DJ, North BV, Sharma R: A novel, externally validated inflammation-based prognostic algorithm in hepatocellular carcinoma: the prognostic nutritional index (PNI). Br J Cancer. 2012, 106:1439-1445. 10.1038/bjc.2012.92

8. Nakatani M, Migita K, Matsumoto S, et al.: Prognostic significance of the prognostic nutritional index in patients with recurrent esophageal squamous cell carcinoma. Nutr Cancer. 2018, 70:467-473. 10.1080/01635581.2018.1445771

9. Watanabe I, Kanauchi N, Watanabe H: Preoperative prognostic nutritional index as a predictor of outcomes in elderly patients after surgery for lung cancer. Jpn J Clin Oncol. 2018, 48:382-387. 10.1093/jico/hyy014

10. Nozoe T, Ninomiya M, Maeda T, Matsukuma A, Nakashima H, Ezaki T: Prognostic nutritional index: a tool to predict the biological aggressiveness of gastric carcinoma. Surg Today. 2010, 40:440-443. 10.1007/s00595-009-4065-y

11. Ren H, Wu L, Hu W, Ye X, Yu B: Prognostic value of the c-reactive protein/prognostic nutritional index ratio after hip fracture surgery in the elderly population. Oncotarget. 2017, 8:61365-61372. 10.18632/oncotarget.18135

12. Hu Q, Wang G, Ren J, et al.: Preoperative prognostic nutritional index predicts postoperative surgical site infections in gastrointestinal fistula patients undergoing bowel resections. Medicine (Baltimore). 2016, 95:4084. 10.1097/MD.0000000000004084

13. Jenckel F, Knecht R: State of the art in the treatment of laryngeal cancer . Anticancer Res. 2013, 33:4701-4710.

14. Chen S, Yang X, Feng JF: A novel inflammation-based prognostic score for patients with esophageal squamous cell carcinoma: the c-reactive protein/prognostic nutritional index ratio. Oncotarget. 2016, 7:62123-62132. 10.18632/oncotarget.11389

15. Manly DA, Boles J, Mackman N: Role of tissue factor in venous thrombosis . Annu Rev Physiol. 2011, 73:515-525. 10.1146/annurev-physiol-042210-121137

16. McSorley MA, Alberg AJ, Allen DS, et al.: C-reactive protein concentrations and subsequent ovarian cancer risk. Obstet Gynecol. 2007, 109:933-941.

17. Hefler LA, Concin N, Hofstetter G, et al.: Serum C-reactive protein as independent prognostic variable in patients with ovarian cancer. Clin Cancer Res. 2008, 14:710-714. 10.1158/10780432.CCR-07-1044

18. Kemik O, Sumer A, Kemik AS, et al.: The relationship among acute-phase response proteins, cytokines and hormones in cachectic patients with colon cancer. World J Surg Oncol. 2010, 8:85. 10.1186/1477-7819-8-85

19. Reynes G, Vila V, Martin M, Parada A, Fleitas T, Reganon E, Martinez-Sales V: Circulating markers of angiogenesis, inflammation, and coagulation in patients with glioblastoma. J Neurooncol. 2011, 102:35-41. 10.1007/s11060-010-0290-X

20. Liu Y, Chen S, Zheng C, et al.: The prognostic value of the preoperative c-reactive protein/albumin ratio in ovarian cancer. BMC Cancer. 2017, 17:285. 10.1186/s12885-0173220-x

21. Ottery FD: Cancer cachexia: prevention, early diagnosis, and management . Cancer Pract. 1994, 2:123-131.

22. Gourin CG, Couch ME, Johnson JT: Effect of weight loss on short-term outcomes and costs of care after head and neck cancer surgery. Ann Otol Rhinol Laryngol. 2014, 123:101-110.

$10.1177 / 0003489414523564$ 


\section{Cureus}

23. Milne K, Alexander C, Webb JR, et al.: Absolute lymphocyte count is associated with survival in ovarian cancer independent of tumor-infiltrating lymphocytes. J Transl Med. 2012, 10:33. 10.1186/1479-5876-10-33

24. Liu B, Huang Y, Sun Y, et al.: Prognostic value of inflammation-based scores in patients with osteosarcoma. Sci Rep. 2016, 6:39862. 10.1038/srep39862

25. Nozoe T, Kimura Y, Ishida M, Saeki H, Korenaga D, Sugimachi K: Correlation of pre-operative nutritional condition with post-operative complications in surgical treatment for oesophageal carcinoma. Eur J Surg Oncol. 2002, 28:396-400. 10.1053/ejso.2002.1257

26. Sun WW, Hu JA, Niu WQ, Gao BL, Xu ZH: Significance of NLDA, the commixed index of inflammation, immune responses, hemostasis, and nutrition, for predicting metastatic nonsmall cell lung cancer prognosis and metastases. Oncotarget. 2017, 8:81978-81993. 10.18632/oncotarget.18184

27. Zhang W, Ye B, Liang W, Ren Y: Preoperative prognostic nutritional index is a powerful predictor of prognosis in patients with stage III ovarian cancer. Sci Rep. 2017, 7:9548. 10.1038/s41598-017-10328-8 